\title{
Evaluation of Aggregatibacter actinomyctemcomitans in periodontitis Patients of Metabolic Syndrome and Periodontitis Patients without Metabolic Syndrome - A Dentistry Study
}

\author{
Ashwini Walke*, Tushar Bhagat, Madhuri Gawande and Minal Chaudhary \\ ${ }^{1}$ Department of Oral Pathology and Microbiology, Riyadh, Saudi Arabia \\ ${ }^{2}$ Department of Prosthodontics, Riyadh, Saudi Arabia \\ ${ }^{3}$ Department Oral Pathology and Microbiology, SPDC, Wardha, Maharashtra, India \\ *Corresponding author
}

A B S T R A C T

Oral cavity is said to be the mirror of systemic health. It has long been known, that many systemic diseases first manifest in the oral cavity. On the other hand oral diseases have a

Keywords potential to act as an independent risk factor for causing systemic disease. Currently, this bidirectional view is quickly gaining acceptance, due to significant findings supporting the

Aggregatibacter actinomyctemcomitans, Metabolic Syndrome, Periodontitis.

\section{Article Info}

Accepted:

12 October 2017

Available Online:

10 December 2017 association between periodontal disease and systemic conditions such as CVD, T2DM, adverse pregnancy outcomes, osteoporosis, Metabolic Syndrome. Significant effort has brought numerous advances in revealing the etiological and pathological links between this chronic inflammatory dental disease and these other conditions. The role of microorganisms in the causation and pathogenesis of periodontal disease is well documented. Within the past 10 years, many studies have been published indicating a positive or negative relationship between periodontitis and various systemic diseases, including Met.S. The most frequently identified periodontal pathogens include three microaerophilic species (A. actinomyctemcomitans, Campylobacter rectus, and Eikenella corrodens). The results of the present study confirmed increased colonization of periodontal pathogen, A. actinomyctemcomitans in both periodontitis patients without Met.S and Periodontitis patients with Met.S.

\section{Introduction}

Oral cavity is an open system exposed to the environment. Furthermore, the possibilities of foreign material entering the system from the oral cavity are heightened due to the constant intake of food and liquids through the mouth. The presence of the large numbers of bacteria can induce tissue destruction indirectly by activating host defense cells, which in turn, produce and release mediators that stimulate the effectors of connective tissue breakdown. ${ }^{1-13}$ According to WHO Report
2008, In India, 53\% of the deaths were due to Non Communicable Diseases (NCD). Cardiovascular disease (CVDs) alone account for 24 percent of all deaths. These NCD include hypertension, heart diseases, stroke, diabetes, obesity, high cholesterol and diseases associated with tobacco use (smoking and chewing) like chronic bronchitis, Chronic obstructive pulmonary disease (COPD), cancer and excessive use of alcohol. Moreover, a substantial proportion of 
these deaths are in the productive age-group and all of them are preventable in nature. But, the rising challenge due to NCDs is that it increasingly affecting the younger populations. ${ }^{2}$ Bacteria inhabits the oral cavity from birth to death. They colonize the soft tissue including the gingiva, cheeks and tongue and when teeth are present, bacteria colonize them even below the gingival margin. It is estimated that between 300 to 400 different bacterial species are capable of colonizing mouth, and any individual may typically harbor 150 to 200 different species. ${ }^{3-}$ 15-16 Bacteria initially colonize and interact with the tooth and then through physical and physiologic interactions among different species with in the microbial mass, there is formation of biofilm and dental plaque. Numerous studies have shown that microbiota of periodontal diseases is significantly different from that of periodontal health.

It is also worth mentioning that different type of periodontal diseases are characterized by a presence of particular group of organisms. ${ }^{4}$ In case of periodontitis high percentage of anaerobic (90\%), gram negative (75\%) bacterial species are present in this type of periodontitis. Bacteria found in the high levels includes $P$. gingivalis, $T$. forsythia, $P$. intermedia, $C$. rectus, $E$. corrodens, $F$. nucleatum, $A$. actinomyctemcomitans, $P$. microns and Treponema and Eubacterium spp. Bacteria found to be elevated in active sites $C$. rectus, $P$. gingivalis, $P$. intermedia, $F$. nucleatum and $T$. forsythia. Detectable level of $P$. gingivalis, $P$. intermedia, $T$. forsythia, $C$. rectus and $A$. actinomyctemcomitans are associated with disease progression. ${ }^{4-14}$ The term periodontitis refers to an inflammatory disease of the supporting tissues of the teeth caused by specific microorganism or group of specific microorganism resulting in progressive destruction of the periodontal ligament and alveolar bone resulting in pocket formation or recession or both.
The clinical cluster of hypertension, cardiovascular disease, hyperlipemia, hyperuricemia and type 2 Diabetes had been recognized by physicians for many decades, described as syndrome $\mathrm{X}$ and finally metabolic syndrome (Met.S) ${ }^{5}$ which was the subjects of this study. Metabolic Syndrome (Met.S) it is a cluster of Cardiovascular risk factor that includes Obesity, Diabetes, Hypertension and Dyslipidemia. Subjects meeting 3 of these criteria were classified as having Met.S. (NCEP, 2001).

There is a known association between periodontitis and Met.S which warrants further investigations. The microorganisms associated with periodontitis are diverse. The association between A.actinomyctemcomitans and periodontitis is well established but the association between A.actinomyctemcomitans and Met.S is not so evident. Studies have also reported presence of $A$. actinomyctemcomitans in periodontitis, but microbiological status in Met.S patients is not yet evaluated. Hence, with this aim in mind, we conducted the present study to evaluate the incidence of A.actinomyctemcomitans in periodontitis patients with Met.S and patients with periodontitis without Met.S.

\section{Materials and Methods}

The present study was carried out in the Department of Oral Pathology and Microbiology of Sharad Pawar Dental College. The patients were selected from the Outpatient Department (OPD) of the department of Periodontics, Sharad Pawar Dental College and department of General Medicine, Achaya Vinoba Bhave Rural Hospital Sawangi, Meghe Wardha. Before the start of the study, an informed consent was obtained from all the study subjects. The study was approved by the Institutional Ethics Committee, Datta Meghe Institute of Medical Sciences, Wardha (Deemed University) Ref. 
No. DMIMS (DU)/ IEC/ 2013-14/ 127 date30.09.2013. The study was performed on subjects, which were divided into three groups according to their periodontal status and presence or absence of Met.S.

Group I: Periodontally healthy subjects without systemic disorder. $(\mathrm{n}=50)$.

Group II: Chronic Periodontitis patients without Met.S ( $\mathrm{n}=50)$.

Group III: Chronic Periodontitis patients with Met.S ( $=50)$

The criteria for periodontal healthy sites were

Probing pocket depth $(\mathrm{PPD}) \leq 3 \mathrm{~mm}$.

No signs of inflammation or mild inflammation.

Absence of bleeding after probing (BOP).

Gingival index (GI) score $\leq 1$.

The criteria for periodontal disease sites were as follows

Probing pocket depth (PPD) $>3 \mathrm{~mm}$, with the help of Williams graduated periodontal probe.

Presence of bleeding on probing (BOP).

Presence of clinical attachment loss (CAL).

Gingival Index score (GI $\geq 2)$

The criteria for diagnosis of Met.S were as follows

Body Mass Index (BMI), Obesity > 30( mass $^{\mathrm{kg}} /$ height $\mathrm{m}^{2}$ )

Pressure $(\mathrm{BP})>140 / 90 \mathrm{mmHg}$
Triglycerides $(\mathrm{TG})>160 \mathrm{mg} \%$

Fasting Blood Sugar $($ FBS $)>125 \mathrm{mg} / \mathrm{dl}$

High -Density Lipoprotein (HDL) > 75mg\%

Detailed clinical history was recorded and clinical assessment was carried out in all the three groups. Subgingival plaque samples were collected and subjected to conventional microbial culture method.

For Group I - Subgingival plaque sample was collected from molar area of periodontally healthy individuals. The sampling site was isolated using cotton rolls and supragingival plaque was removed with the help of sterile cotton. The subgingival plaque sample was then collected using sterile Gracey curettes. For Group II and III- The sampling sites were isolated with cotton roles and subgingival plaque was removed using sterile cotton. Subgingival plaque samples were obtained using sterile gracy curettes from deepest periodontal pocket. Plaque was subsequently transferred into a sterile container (Himedia) with $0.85 \%$ of sterile saline that was immediately processed. Serial Dilution was done. A serial dilution is the stepwise dilution of a substance in solution. Usually the dilution factor at each step is constant, resulting in a geometric progression of the concentration in a logarithmic fashion. In 100 $\mathrm{ml}$ distilled water, $0.85 \mathrm{gms}$ Sodium chloride ( $\mathrm{NaCl}$ - SD fine company) was added. The prepared $9 \mathrm{ml}$ of $0.85 \% \mathrm{NaCl}$ was dispensed into 3 test-tubes (Borosil) each and autoclaved. After autoclaving 5-fold serial dilutions of the sample was made in the testtube containing $0.85 \% \mathrm{NaCl}$. The serial dilution of samples were done immediately and cultured on agar plates. Tryptic SoySerum Bacitracin Vancomycin Agar is recommended for the isolation of $A$. actinomyctemcomitans (Table 1 and 2). The prepared culture plate was used for 
inoculation. The plaque sample which was already serially diluted was added to each medium using streak culture method. Streak culture method is a routinely employed method for bacterial isolation in pure culture. A platinum or nichrome wire loop of 2-4 nm in internal diameter is used. A loopful of specimen is smeared onto the surface of dried plate near the peripheral area. This is known as primary inoculum. From the primary inoculum, it is spread thinly over the plate by streaking with loop in parallel lines. The streak plate technique is essentially a method to dilute the number of organisms, decreasing the density. This allows for individual colonies to be isolated from other colonies. Each colony is considered "pure," since theoretically, the colony began with an individual cell. Each inoculated petri-plate was sealed with Parafilm (Himedia) to prevent any contamination while incubation, and then placed in an anaerobic jar (Himedia) with AnaeroGas Pack system (Himedia) and incubated at $37^{\circ} \mathrm{c}$ for 48 hours for $A$. actinomyctemcomitans. All the plates are then placed in the anaerogas jar and anaerogas pack is placed inside the jar to maintain the anaerobic condition. The anaerobic gas jar is then placed inside the incubator at $37^{0} \mathrm{C}$ for 72 hours. After 72 hours the colonies cultured on Petri plates are subjected to biochemical identification test for confirmation of bacteria intended to be cultured. The identification of a bacterial species is based on factors, including colony morphology, chemical composition of cell walls, biochemical activities, and nutritional requirements. 3 points should be considering during microorganism identification (Table 2). A. actinomyctemcomitans is gram negative and catalase test and Indole Test were positive. Numbers of colonies of bacteria grown on each plate were counted by colony counter. By working backwards using multiplication with 'dilution factor' (the number of times that you have diluted the bacteria sample with diluents solution), the number of bacteria of the original sample can be determined. To compute the estimated number of bacteria, the following formula was used. $B=N / D$, $\mathrm{B}=$ number of bacteria, $\mathrm{N}=$ number of colonies counted on a plate, $\mathrm{D}=$ dilution factor $(1,10$ or 100).

\section{Results and Discussion}

In this study, a quantitative analysis of $A$. actinomyctemcomitans in periodontitis patients with Met.S and periodontitis patients without Met.S done. The results of the present study were subjected to statistical analysis. The comparison of the bacterial counts in periodontitis patients with Met.S (Group III), periodontitis patients without Met.S (Group II) and normal control (Group I) was carried out to find the significant difference between those values. The statistical tests used for the analysis of the results were: Chi-square Test, One way ANOVA (F-Test), Tukey HSD and 'Descriptive statistical analysis' (i.e. mean, standard deviation and standard error) was carried out for all the groups in this study.

Among 150 selected patients, $A$. actinomyctemcomitans were present in 2(4\%) patients of normal group, 26(52\%) patients of periodontitis without Met.S, and 33(66\%) patients of periodontitis with Met.S. Therefore, the results of the study revealed that, A. actinomyctemcomitans is present in descending order from Group III $>$ Group II $>$ Group I (Table 3A). Among all groups, $A$. actinomyctemcomitans were present in $1(50 \%)$ male and $1(50 \%)$ female patient of normal group, $14(53.84 \%)$ male patients and $12(46.15 \%)$ female patients of periodontitis without Met.S (Group II), 26(78.78\%) male and $7(21.21 \%)$ female patients of periodontitis with Met.S (Group III). Presence of A. actinomyctemcomitans in males was more as compared to females in group II and group III, and there was 4:1 distribution of 
male: female in Group III and interpreted in Table 3B. In Normal controls (Group I), mean total count for $A$. actinomyctemcomitans was $0.06 \pm 0.31 \times 10^{5}$ with range $0.00-2.00$. In Periodontitis patients without Met.S (Group II), mean total count for $A$. actinomyctemcomitans was $3.76 \pm 3.86 \times 10^{5}$ with range $0.00-10.00$. In Periodontitis patients with Met.S (Group III), mean total count for $A$. actinomyctemcomitanswas $6.56 \pm 5.45 \times 10^{5}$ with range $0.00-20.00$. Thus, mean count of A.actinomyctemcomitans in periodontitis patients with Met.S was three times more when compared to patients with periodontitis without Met.S. In periodontitis patients without Met.S the count was about three times more than normal control. In periodontitis patients with Met.S the count was about six times more than normal control (Table 4, Graph 1). The statistically significant variations of mean of total count of A. actinomyctemcomitans were found among all groups $(\mathrm{p}=0.000)$. The total counts of A.actinomyctemcomitans were observed in decreasing order in periodontitis with Met. S (Group III) $6.56 \times 10^{5} \pm 5.45 \times 10^{5}$, periodontitis

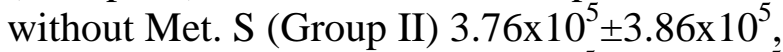
normal control (Group I) $0.06 \times 10^{5} \pm 0.31 \times 10^{5}$ (Table 5). The statistical significant difference in total count $A$. actinomyctemcomitans was noted between all groups $(\mathrm{p}=0.000)$. The statistical analysis revealed that count of $A$. actinomyctemcomitans was highest in periodontitis patients with Met.S (Group III) than periodontitis patients without Met.S (Group II) and normal control (Group I) (Table 6).

The most frequently identified periodontal pathogens include three microaerophilic species (A. actinomyctemcomitans, Campylobacter rectus, and Eikenella corrodens) and seven anaerobic species $(P$. gingivalis, Bacteroides forsythus, $T$. denticola, Prevotella intermedia, Fusobacterium nucleatum, Eubacterium, and spirochetes). Socransky et al., divided the pathogens into two main clusters and deemed them the "red" and "orange" complexes. Furthermore, they defined "green", "yellow", and "purple" complexes as the bacterial colonies that formed on the tooth surface prior to the colonization of the "orange" and "red" complexes respectively. The "red" complex consisted of three tightly related species: $T$. forsythensis, $P$. gingivalis and $T$. denticolaand were thought to be most pathogenic to humans. ${ }^{6}$

It is known that all Met.S triggering factors play an important role in the onset of oxidative stress, subsequent formation of ROS, and probably also in the activation of the pro-oxidising, pro-inflammatory AGERAGE system. Many inflammatory pathways are activated by these conditions. The excess of visceral fat (high waist circumference) is certainly one of the most important factors in activating these signalling molecular cascades through the TNF-alfa pathway. The activation of these pathways is not restricted to limited areas of the body, but their signalling triggers systemic responses, which are also visible at the level of teeth supporting tissues. ${ }^{7}$

Our study results revealed that, the mean count of A.actinomyctemcomitans in periodontitis patients was four times more than normal control. The findings of our study were in accordance with the results of the study by Slots et al., 1980; Mandell and Socransky, 1981. Who documented a rise of A. actinomycetemcomitans in periodontitis patients than in healthy individuals. ${ }^{8-}$ ${ }^{9}$ A.actinomyctemcomitans present in the periodontal pocket was associated with preadolescent, localized juvenile and advanced adult aggressive periodontal disease. Several virulence factors are associated with it. Leukotoxin is the most important. 
Table.1 Composition of tryptic soy-serum bacitracin vancomycin agar

\begin{tabular}{|c|l|l|}
\hline Sr. No. & \multicolumn{1}{|c|}{ Ingredients } & \multicolumn{1}{c|}{ Grams/ Litre } \\
\hline 1. & Tryptic Soy Agar & $40.0 \mathrm{~g}$ \\
\hline 2. & Yeast Extract & $1.0 \mathrm{~g}$ \\
\hline 3. & Bacitracin & $75.0 \mathrm{mg}$ \\
\hline 4. & Vancomycin & $5.0 \mathrm{mg}$ \\
\hline 5. & Horse Serum & $100.0 \mathrm{ml}$ \\
\hline 6. & Distilled Water & $1000.0 \mathrm{ml}$ \\
\hline
\end{tabular}

Final $\mathrm{pH}\left(\right.$ at $\left.25^{\circ} \mathrm{C}\right) 7.1 \pm 0.2$

Table.2 Colony morphology (from agar plates)

\begin{tabular}{|c|l|l|c|c|}
\hline Shape & \multicolumn{1}{|c|}{ Elevation } & Edge & Color & Surface \\
\hline Small, star like & Raised & Irregular & Transparent to light & Smooth \\
\hline
\end{tabular}

Table.3A Presence of A. actinomyctemcomitans colonies group wise

\begin{tabular}{|l|c|c|c|c|c|}
\hline Groups & Present & Absent & Total & *2-value \pm SD & p-value \\
\hline Group I & $02(4 \%)$ & $48(96 \%)$ & $50(100 \%)$ & & \multirow{2}{*}{$\mathrm{p}<0.01$} \\
\cline { 1 - 5 } Group II & $26(52 \%)$ & $24(48 \%)$ & $50(100 \%)$ & $7.23 \pm 1.17$ & \\
\hline Group III & $33(66 \%)$ & $17(34 \%)$ & $50(100 \%)$ & $9.94 \pm 3.30$ & \\
\cline { 1 - 5 } Total & 61 & 89 & 150 & & \\
\hline
\end{tabular}

Table.3B Distribution of A. actinomyctemcomitans according to gender in different groups

\begin{tabular}{|l|l|l|l|}
\hline Gender & Group I & Group II & Group III \\
\hline Male & $01(50 \%)$ & $14(53.84 \%)$ & $26(78.78 \%)$ \\
\hline Female & $01(50 \%)$ & $12(46.15 \%)$ & $7(21.21 \%)$ \\
\hline Total & $02(100 \%)$ & $26(100 \%)$ & $33(100 \%)$ \\
\hline
\end{tabular}

Comparison of total count for A.actinomyctemcomitans in three groups (all the values in $10^{5}$ )

Table.4 Descriptive Statistics for A.actinomyctemcomitans in three groups (All the values in $10^{5}$ )

\begin{tabular}{|c|c|c|c|c|c|c|c|c|}
\hline & & & \multirow{2}{*}{ Groups } & N & Mean & $\begin{array}{c}\text { Std. } \\
\text { Deviation }\end{array}$ & $\begin{array}{c}\text { Std. } \\
\text { Error }\end{array}$ & \multicolumn{2}{|c|}{$\begin{array}{c}\text { Interval for Mean } \\
\text { Inter }\end{array}$} & \multirow{2}{*}{$\begin{array}{c}\text { Lower } \\
\text { Bound }\end{array}$} & $\begin{array}{c}\text { Upper } \\
\text { Bound }\end{array}$ & & Maximum \\
\hline Normal & 50 & 0.06 & 0.31 & 0.04 & -0.02 & 0.14 & 0.00 & 2.00 \\
\hline $\begin{array}{c}\text { Periodontitis } \\
\text { without Met.S }\end{array}$ & 50 & 3.76 & 3.86 & 0.54 & 2.66 & 4.85 & 0.00 & 10.00 \\
\hline $\begin{array}{c}\text { Periodontitis } \\
\text { with Met.S }\end{array}$ & 50 & 6.56 & 5.45 & 0.77 & 5.01 & 8.10 & 0.00 & 20.00 \\
\hline
\end{tabular}


Table.5 One way ANOVA for A. actinomyctemcomitans in three groups (All the values in $10^{5}$ )

\begin{tabular}{|c|c|c|c|l|c|}
\hline Source of variation & Sum of Squares & Df & Mean Square & F & p-value \\
\hline Between Groups & 1063.00 & 2 & 531.56 & & \multirow{2}{*}{0.000} \\
\hline Within Groups & 2192.26 & 147 & 14.91 & 35.63 & S,p $<0.05$ \\
\hline Total & 3255.26 & 149 & & & \\
\hline
\end{tabular}

Table.6 Multiple comparisons: Tukey Test for A. actinomyctemcomitans in three groups (All the values in $10^{5}$ )

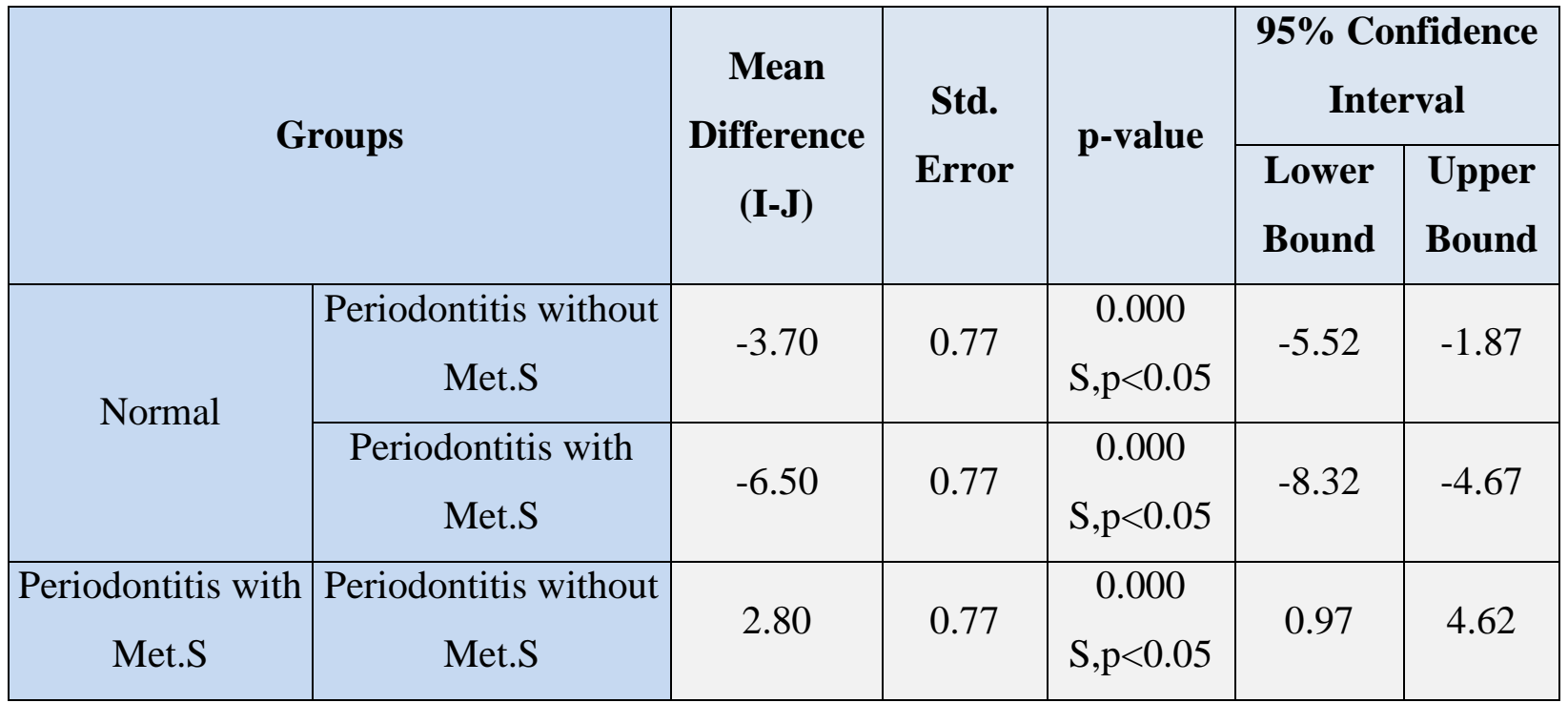

Graph.1 Comparison of total count for A. actinomyctemcomitans in three groups (All values in $10^{5}$ )

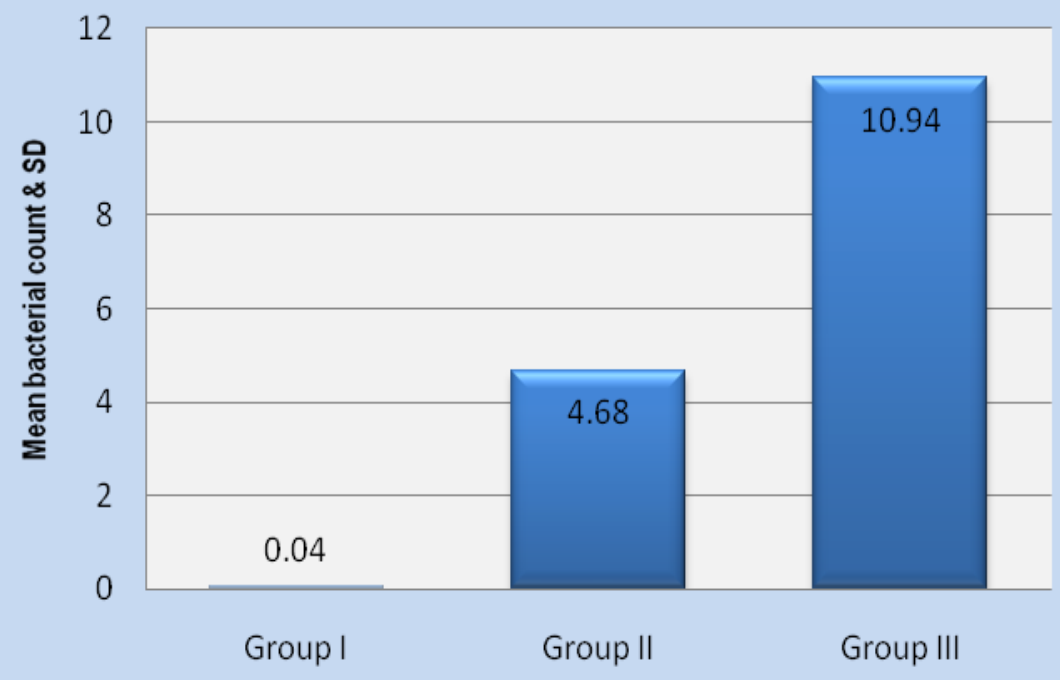


Graph.3A Presence of A. actinomyctemcomitans colonies group wise

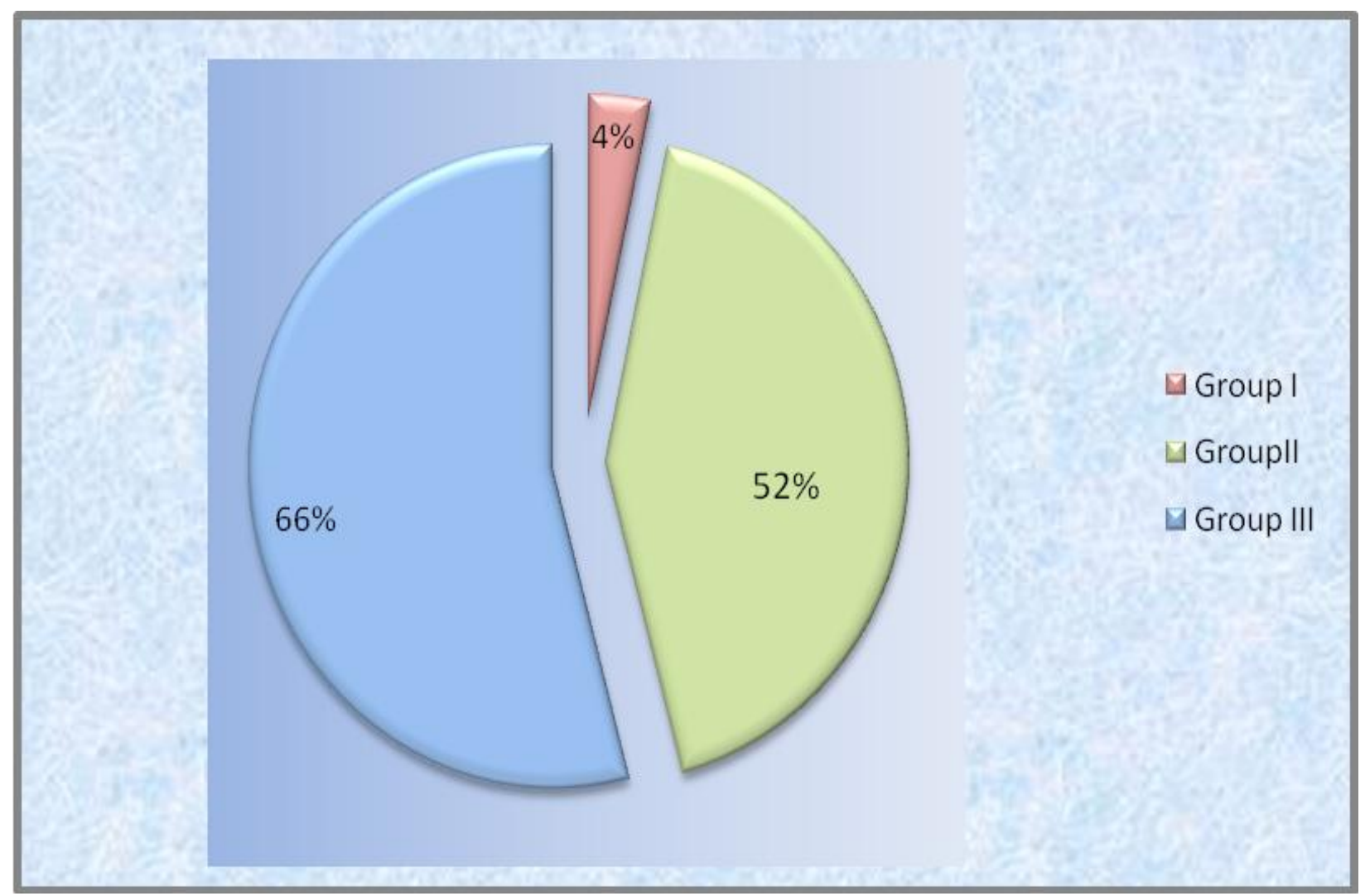

Fig.1 A. actinomyctemcomitans Growth in (A) Group I, (B) Group II, (C) Group III

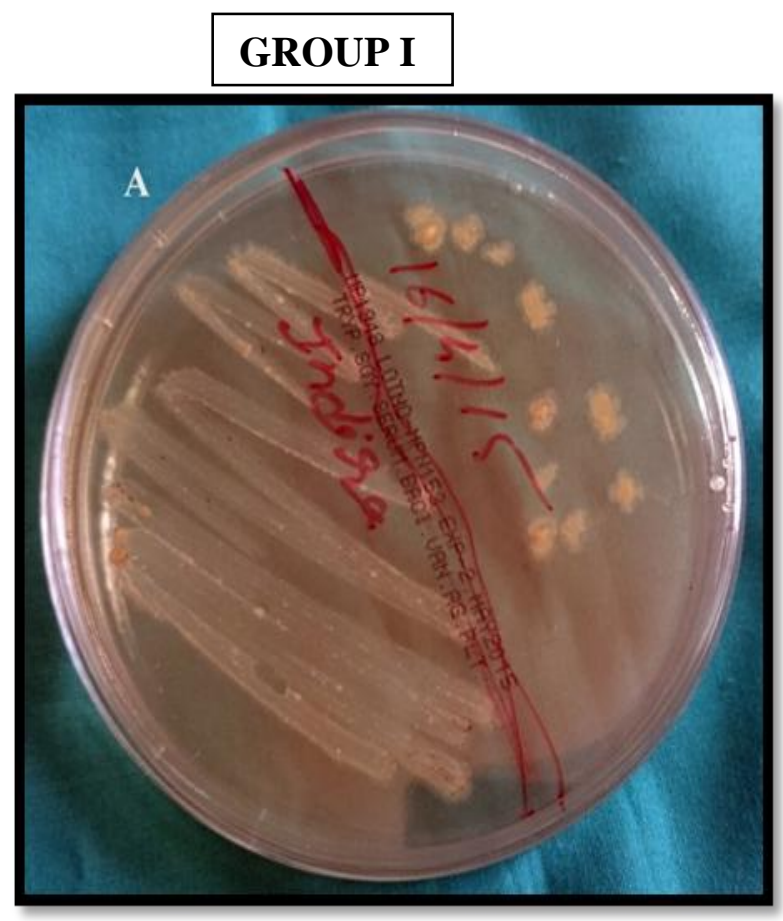

\section{GROUP II}

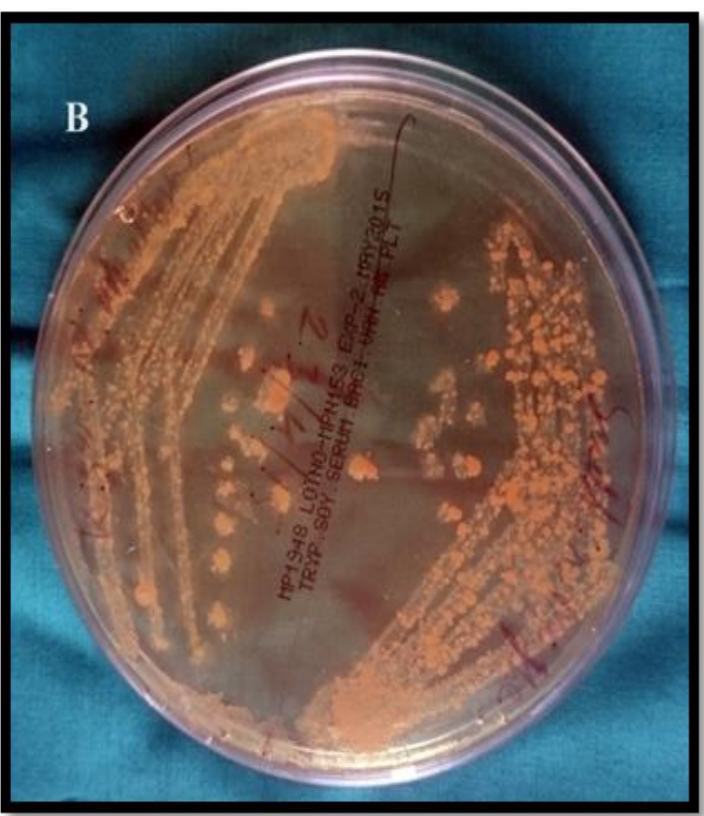




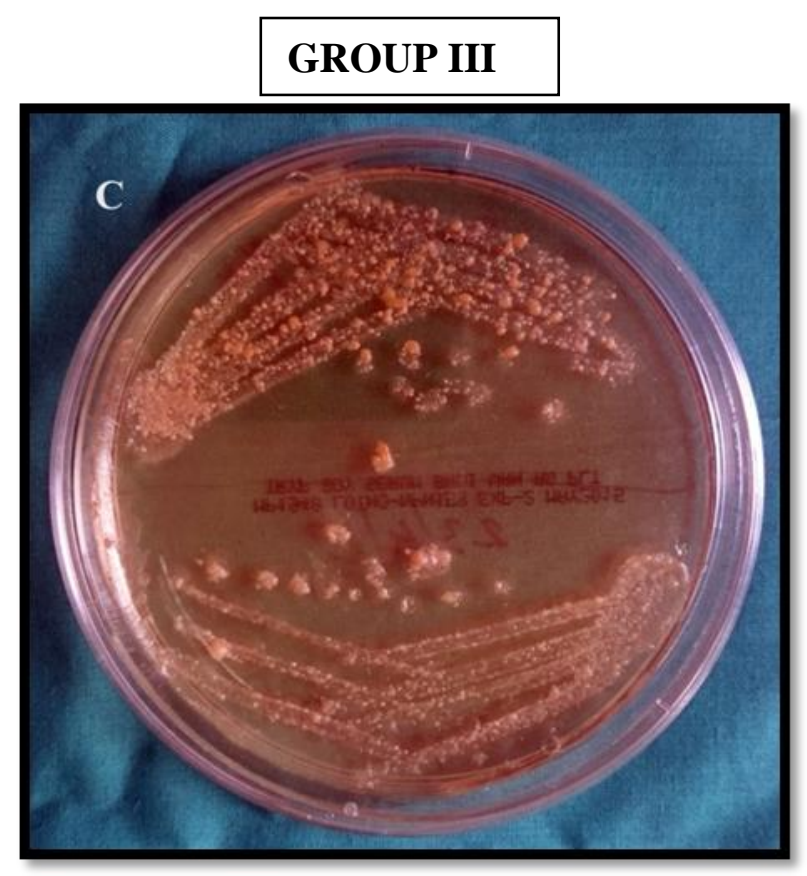

Colour Plates-I

Also cytolethal distending toxin, immunosuppression factors and inhibition of PMNS functions are also seen. Leukotoxin from A. actinomyctemcomitans can kill human and non-human primate polymorphonuclear leukocytes and peripheral blood monocyte that are the innate immune response and these could be attacked directly. A. actinomycetemcomitans endotoxin has the potential to modulate host responses and contribute to tissue destruction. The ability of the A. actinomyctemcomitans lipopoly saccharide to stimulate macrophages to release interleukin IL-1, IL-1 $\beta$, and TNF is of significance. These cytokines are capable of stimulating bone resorption and thereby contributing to periodontitis. ${ }^{10}$

Our study results also revealed that the mean count for A. actinomyctemcomitans in periodontitis patients with Met.S was three times more than periodontitis patients without Met.S. and double than normal control. The results of our study were in accordance with the study done by Sakalauskiene et al., (2014) who reported increased in
A.actinomyctemcomitans in periodontitis patients with Met.S.

The cause of increase in A.actinomyctemcomitans in periodontitis with Met.S could be, T2DM and increased BMI which are components of Met.S. A.actinomyctemcomitanswas significantly and positively correlated to BMI, and the prevalence of the periodontal pathogen A.actinomyctemcomitans was significantly higher in diseased sites than in healthy sites in both type 1 and type 2 diabetes patients. In obesity the increased inflammation, favors oral microbes to disrupt the endocrine function of the adipose tissue which in turn would cause an imbalance in glucose homeostasis. Thus it could be hypothesized that, increased levels of leptin aggravates periodontitis and will contributes to increased in colonies of A,actinomyctemcomitansin periodontitis patients with Met.S. ${ }^{11}$

Growth favoring conditions in Met.S could be- A.actinomyctemcomitans requires Lcystein, iron and fat soluble vitamins for their 
growth, ${ }^{12}$ which are thought to be provided in Met.S patients that could be responsible for increases in number of colonies in Met.S patients. However, there is lack of data available regarding this. Also it could be hypothesized that in Met.S both innate and acquired immunity are altered, which likely to be contributing factor of increase in initial colonization of A.actinomyctemcomitans.

The results of the present study confirmed increased colonization of periodontal pathogen, A.actinomyctemcomitans in both periodontitis patients without Met.S and Periodontitis patients with Met.S. The fact that increases in colonization of periodontal pathogens may be attributed to the assumption that, the common link between periodontitis and Met.S is the action of ROS either acting as a second messenger or directly damaging target molecules as proteins, lipids or DNA. Periodontitis releases proinflammatory cytokines and ROS at the site of inflammation, leading to oxidative stress situation. ${ }^{13}$ This will contribute to aggravate existing Met.S. both of which act synergistically is a matter of debate.

Thus, Researchers have hypothesized about the etiologic role of systemic diseases in the pathogenesis of periodontitis. Patients diagnosed with Met.S are thought to be at higher risk due to a compromised immune system. Infectious and opportunistic microbes responsible for periodontal infection may thus bring a burden onto the rest of the body. Furthermore, these microbes can release products that elicit an inflammatory response. Systemic conditions are recognized as continually renewing reservoirs for the periodontitis producing bacterial antigens, Gram-negative bacteria, cytokines, and other proinflammatory mediators.

Therefore, Met.S subjects should be recommended to go for frequent screening and periodontal treatment. Also, chronic periodontitis patients should be considered for multidisciplinary approach by physician, bearing in mind that the periodontal tissues are exposed not only to local bacterial onslaught, but also systemic conditions damaging them through the same mechanisms provoking damage in other tissues. To control the severity of Met.S and restrict the morbidity and mortality attributed to the components of Met.S should be the combined aim of the physician and dentist.

\section{Acknowledgment}

The first author would like to acknowledge Dept. of Oral Pathology and Microbiology, Dept. of Periodontics, Sharad Pawar Dental College and department of General Medicine, Achaya Vinoba Bhave Rural Hospital Sawangi, Meghe Wardha.

\section{References}

1. Jemin Kim and Boston. Periodontal disease and systemic conditions: a bidirectional Relationship. NIH Odontology. September 2006; 94(1): 1021.

2. Joy Kumar Chakma, Sanjay Gupta. Review Article. Lifestyle and NonCommunicable Diseases: A double edged sword for future India. Indian Journal of Community Health, Oct - Dec 2014, Vol 26, Issue no 04.

3. Paster BJ, Boches SK, Galvin JL, Ericson RE, Lau CN, Levanos VA et al., Bacterial diversity in human subgingival plaque. J. Bacteriol 2001;183;3370-83.

4. Newman MG, Takei HH, Carranza FA. Carranza's Clinical Periodontology. $9^{\text {th }}$ ed. Philadelphia: Saunders; 2003.

5. Aye: Metabolic syndrome, J Metabolic syndrome 2014:3;4.

6. Maria Grazia Cifone, Annalisa Monaco, Davide Pietropaoli, Rita DelPinto and 
Mario Giannoni. Advanced Glycation End Products: Possible link Between Metabolic Syndrome and Periodontal Diseases. Pathogenesis and treatment of periodontitis. Pp. 97-108.

7. Mandell RL, Socransky SS. A selective medium for Actinobacillus actinomycetemcomitans and the incidence of the organism in juvenile periodontitis. J Periodontol. Oct 1981; 52(10): 593-8.

8. R L Mandell. A longitudinal microbiological investigation of Actinobacillus actinomycetemcomitans and Eikenella corrodens in juvenile periodontitis. Infect Immun. 1984 Sep; 45(3): 778-780.

9. Ljiljana Kesic1, Jelena Milasin2, Marija Igic3, Radmila Obradovic. Microbial Etiology of Periodontal Disease - Mini Review. FACTA Universitatis Series: Medicine and Biology 2008,Vol.15, No 1 pp. $1-6$.

10.Gregor and Hotamisligil, 2011; Teeuw, Gerdes and Loos, Periodontal Microorganisms, Obesity, Chronic Inflammation. University of South Carolina Scholar Commons Theses and Dissertations 12-15-2014.

11. Sreenivasan PK, Meyer DH, Fives- Taylor PM. Factors influencing growth and viability of A. actinomycetemcomitance. Oral Microbial Immunol. Dce 1993, 8(6): 361-9.

12. Sergio Granados, Nuri-El-Azem, Jose L. Quiles, Patrica Perez-Lopez, Adrian Gonzalez, and MCarmen RamirezTortosa. Relationsip between cardiovascular risk factor and periodontal disease: Current Knowledge. Pp. 193-216.

13. Priyalakshmi S, Sankari M. A Review on Metabolic Syndrome and Periodontal disease. J. of Dental and Medical Sciences. Jan 2014. 13(1) ver.IV: 01-05.

14. Young-Eun Kwon, Jung-Eun Ha,, Dai-Il Paik, Bo-Hyoung Jin, Kwang-Hak Bae. The relationship between periodontitis and metabolic syndrome among a Korean nationally representative sample of adults. J Clin Periodontol 2011; 38: 781-786.

15. Soncransky SS, Haffajiee AD. Evidence of bacterial etiology: a historical perspective. Periodontol 2000 1994:5;7-25.

16. Page RC, Offenbacher S, Shroeder HE. Advances in pathogenesis of periodontitis: summary of developments, clinical implications and future directions. Periodontol 2000 1997: 14: 216-28.

\section{How to cite this article:}

Ashwini Walke, Tushar Bhagat, Madhuri Gawande and Minal Chaudhary. 2017. Evaluation of Aggregatibacter actinomyctemcomitans in Periodontitis Patients of Metabolic Syndrome and Periodontitis Patients without Metabolic Syndrome - A Dentistry Study. Int.J.Curr.Microbiol.App.Sci. 6(12): 1685-1695. doi: https://doi.org/10.20546/ijcmas.2017.612.190 